questionnaires on their socio-demographic, reproductive, family and medical history, and behaviours/exposures such as dental and surgical procedures, blood transfusion, induced abortion, early sexual exposure, multiple sexual partners, anal sex, acupuncture, ear and body piercing, body tattoo and sharing needles for drug use.

Results Sixteen of 1105 (1.4\%, 95\% CI 1.0 to 2.0\%) mothers test positive for HBV infection. The most common behaviours/exposures were ear piercing $(77.5 \%)$, dental procedures $(51.2 \%)$, surgical procedures $(21.4 \%)$, and other body piercing (12.4\%). Very few reported blood transfusion (3.4\%), body tattoo (3.1\%), induced abortion $(2.9 \%)$, multiple sexual partners $(1.6 \%)$, anal sex $(0.5 \%)$, drug addiction $(0.4 \%)$ or needle sharing $(0 \%)$. The prevalence of HBV infection were significantly higher among confirmed HBV carriers, and those with positive family history of HBV infection, and jaundice. There were no significant differences in HBV infection by ethnicity, history of surgical and dental procedures, history of blood transfusion, or any of the risk behaviours explored.

Conclusion Prevalence of HBV infection was low at $1.4 \%$. Risk behaviours were low due to under reporting or antenatal mothers are lower risk compared to the general population. We did not find any significant association between HBV infections and the explored risk behaviours.

\section{P1-517 DRIVERS AND VISION: A SURVEY IN KERMAN PROVINCE (SOUTHEASTERN PART OF IRAN)}

doi:10.1136/jech.2011.142976h.5

${ }^{1} \mathrm{~A}$ Sharifi, ${ }^{2} \mathrm{H}$ Sharifi, ${ }^{3} \mathrm{H}$ R Esmaili, ${ }^{3} \mathrm{E}$ Daneshtalab. ${ }^{1}$ Department of Ophthalmology, Kerman University of Medical Science, Kerman, Iran; ${ }^{2}$ Department of Food Hygiene and Public Health, Faculty of Veterinary Medicine, Shahid Bahonar University of Kerman, Kerman, Iran; ${ }^{3}$ Kerman University of Medical Science, Kerman, Iran

Introduction About $90 \%$ of sensory information to a driver is through vision. Vision is the most important sense for driving. The purpose of this study was to determine vision and causes of visual defects among public vehicle drivers.

Methods In this cross-sectional study, vision and causes of visual deficits were evaluated among drivers that were selected sequentially by transportation organization in Kerman province in 2009.

Results Of 4000 public drivers assessed, 361 (9.02\%, 95\% CI 9.01 to 9.03) had visual acuity at the level not approved for driving by traffic rules in Iran (with old glasses). After ocular examination and correction of refractive errors they were 69 (1.72\%). Legal blindness $(\mathrm{VA}<1 / 10)$ was found in 13 eyes. The common causes of visual deficits were refractive errors $(88.7 \%)$, age related macular degeneration $(10.9 \%)$ and amblyopia (6.3\%).

Conclusion A large number of public vehicle drivers did not have vision at the level approved for driving in Iran. Most of them, their vision can be improved. Some of them should not be allowed to drive due to irreversible poor vision.

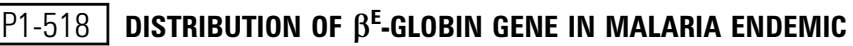 AND NON-ENDEMIC ZONES OF ASSAM, INDIA}

doi:10.1136/jech.2011.142976h.6

S K Sharma, ${ }^{*}$ L Chaliha, J Mahanta. Regional Medical Research Centre, N. E. Region (Indian Council of Medical Research), Dibrugarh, Assam, India

Introduction $\beta^{\mathrm{E}}$-globin gene is the major variant haemoglobin prevalent among the autochthonous population of Assam, India, with variable gene frequencies. The gene frequency for this variant haemoglobin is as high as 0.6 in some of the ethnic groups. The geoclimatic condition of the area also facilitates transmission of Plasmodium falciparum in this part of the country. Distribution pattern of
$\beta^{\mathrm{E}}$-globin gene among autochthonous inhabitants inhabiting in malaria endemic and non-endemic zones was evaluated.

Methods Blood sample were collected from Kachari communities (Bodo \& Mech) inhabiting in malaria endemic $(n=669)$ and nonendemic zones $(n=202)$ adopting stratified random sampling method. Individual samples were screened for Red Cell Indices by automated haematology cell counter and HPLC based Variant Haemoglobin Testing System were used for the detection of variant haemoglobins and thalassaemias.

Results Red Blood Cell indices indicated lower level of haemoglobin, Mean Cell Volume (MCV) and Mean Cell Haemoglobin (MCH) in subject carrying $\beta^{\mathrm{E}}$-globin gene. Gene frequency of $\beta^{\mathrm{E}}$-globin gene in malaria endemic and non endemic zone was 0.586 and 0.483 respectively. However, distribution of $\beta^{\mathrm{E}}$-globin gene is increased in malaria endemic zone. In malaria endemic zone, $\mathrm{Hb}$ F level in subject carrying $\beta^{\mathrm{E}}$-globin gene was significantly differed from non-endemic zone.

Conclusion Significant difference of distribution pattern of $\beta^{\mathrm{E}}$-globin gene and higher level of $\mathrm{Hb} \mathrm{F}$ in subject carrying $\beta^{\mathrm{E}}$ - globin gene in malaria endemic zone is the striking outcome of the study.

\section{P1-519 ASSOCIATION OF SERUM FERRITIN AND TRANSFERRIN SATURATION WITH ALL-CAUSE AND CARDIOVASCULAR DISEASE MORTALITY: NHANES III FOLLOW-UP STUDY}

doi:10.1136/jech.2011.142976h.7

1J Shin, ${ }^{2} \mathrm{D}$ H Lee, ${ }^{2} \mathrm{H}$ G Son. ${ }^{1}$ Prevention \& Management Center, Regional Cardiocerebrovascular Disease Center, Kyungpook National University Hospital, Daegu, Republic of Korea; ${ }^{2}$ College of Medicine, Kyungpook National University, Daegu, Republic of Korea

The purpose of this study is to examine the association between serum ferritin and transferrin saturation with all-cause, and CVD mortality among 13858 persons (men: 6532, women: 7326) aged 20 years and older from death certificate data linked to the NHANESIII of a nationally representative sample of the noninstitutionalized USA population. Serum ferritin and transferrin saturation levels were categorised according to sex-specific quintiles. RR and $95 \%$ CIs were calculated from Cox proportional hazards regression models adjusted for age, race-ethnicity, poverty index, education, body mass index, smoking, alcohol intake, systolic blood pressure, total cholesterol, and Charlson Comorbidity Index. There were no statistically significant associations between serum ferritin and allcause, and CVD mortality. There were statistically significant ushaped associations between transferrin saturation and all-cause mortality in men (first quintile vs third quintile, RR 0.73 (95\% CI 0.61 to 0.88 ), first quintile vs fifth quintile, RR 0.79 (95\% CI 0.65 to 0.95 ) and between transferrin saturation and CVD mortality in women (first quintile vs fourth quintile, RR 0.58 (95\% CI 0.48 to 0.84 ), first quintile vs fifth quintile, RR 0.68 (95\% CI 0.48 to 0.98 ), all tests for trend, $\mathrm{p}<0.01)$. In this large cohort, there was consistent evidence of increasing risk of mortality at lower transferrin saturation levels. In fact, lower transferrin saturation levels were associated with an increased risk of all-cause and CVD mortality. The results are compatible with the possibility that there is an inverse association between transferrin saturation levels and risk of mortality.

\section{P1-520 PREVENTIVE HEALTH OF INVISIBLE AND UNCONTACTED TRIBES IN AMAZON FOREST}

doi:10.1136/jech.2011.142976h.8

1J A Simas, * ${ }^{2} \mathrm{~L}$ L A S Bulcao. ${ }^{1}$ Furnas eletrobras furnas, Rio de Janeiro, Brazil; ${ }^{2}$ Fundação oswaldo cruz, Rio de Janeiro, Brazil

The Amazon has uncontacted indigenous peoples in its forests. To remain away from contemporary civilisation these tribes may be 
interpreted as the invisible population of the forests. Indian uncontacted is a legal concept that defines human populations from pre-Columbian culture that have remained geographically and culturally distant from the western population. Its demographic composition are unknown, registering only some evidence of their existence and meagre or no evidence of their material culture, customs and languages (Azanha, Gilberto. FUNAI-Mimeo, 2006). The Indian Protection Service of National Indian Foundation-SPI/ FUNAI finds that between 1910 and 1957, of the 105 known isolates indians, 72 were contacted and one third of those contacted were extinct. It is estimated that currently about 33 indigenous peoples remain uncontacted. At the time, especially measles and epidemic influenza were the main causes of the extermination of tribes contacted, currently the diseases that most affect the indigenous population are malaria and hepatitis. (Ribeiro, Darcy, 1996). The study describes the current situation and discusses strategies for preventing and controlling diseases in areas inhabited by these Indians in Brazil. The method establishes the parameters of health risks and establishing a surveillance system for the actions of prevention and control of these risk factors to health in these areas.

\section{P1-521 IO IN CHILDHOOD AND LATER RISK OF ALCOHOL-RELATED DISEASE AND DEATH}

doi:10.1136/jech.2011.142976h.9

\section{S Sjölund, ${ }^{*}$ T Hemmingsson, P Allebeck. Karolinska Institutet, Stockholm, Sweden}

Introduction While much research has been performed on associations between intelligence and several different health outcomes, there is as yet no conclusive evidence regarding the association between intelligence and alcohol-related disease and death. Furthermore, most studies have been performed on men. The aim of this study is to further investigate the association between IO and alcohol-related morbidity and mortality and to explore if there are any differences between men and women in this regard.

Method 43980 Swedish subjects (men and women) born 1948, 1953, 1967, 1972 and 1977 who completed an IQ test at age 13 were followed until 2005. Information on alcohol-related disease and death (ICD-diagnoses) has been collected from the National Patient register and the National Cause of Death register, respectively. Adjustments are made for parental socioeconomic position, age, income, education and occupation, obtained from national censuses. ORs for alcohol related disease and death will be estimated using Cox regression models.

Results So far, unadjusted results have shown, in general, a graded reduction in rates of alcohol-related death and disease by increasing IO. This was found both for men and as for women. Results from the Cox regression will be presented, crude and adjusted for both men and women.

Conclusion This study will contribute to the research investigating the association between IQ and alcohol-related disease and death. It is of particular interest to analyse this association also in women.

\section{P1-522 CLUSTERING OF HEALTH BEHAVIOURS IN SCOTLAND: A COMPARISON BETWEEN EAST AND WEST SCOTLAND AND BETWEEN URBAN AND RURAL REGIONS}

doi:10.1136/jech.2011.142976h.10

J Smith, ${ }^{*}$ R Mitchell. University of Glasgow, Glasgow, UK

Introduction There is evidence that health behaviours cluster together in certain patterns and that this varies by individual and socio-demographic characteristics. This study took a different perspective by investigating whether the clustering of health behaviours varies geographically in Scotland.

Methods Secondary, cross-sectional data from the Scottish Health Survey (2003) were analysed. The prevalence of meeting national recommendations on four health behaviours-alcohol, smoking, physical activity and fruit and vegetable consumption-was calculated. Two geographical divisions were made-east and west, and urban and rural. The number of healthy behaviours individuals have was analysed geographically, using binary logistic regression to control for confounders. Clustering was investigated using observed to expected ratios for each pattern of health behaviour, a method which indicates which patterns occur more or less frequently than would be expected by chance.

Results Prevalence of healthy behaviour differed significantly across both geographical divisions, except for alcohol behaviour. After controlling for age, sex and deprivation, a significant but small difference in number of healthy behaviours was found between urban and rural areas, but not between east and west. The most prevalent pattern of behaviour was not drinking or smoking, but also not eating enough fruit and vegetables and not exercising enough. Clustering of health behaviours did not differ geographically.

Conclusion Clustering of health behaviour may not contribute to geographical health inequalities in Scotland. However, the occurrence of multiple unhealthy behaviours was common and may be an important consideration for public health programmes in Scotland.

\section{P1-523 SOCIOECONOMIC-RELATED INEQUALITY IN ORAL HEALTH RISK BEHAVIOURS AMONG ADOLESCENTS IN THAILAND}

doi:10.1136/jech.2011.142976h.11

T Somkotra.* Chulalongkorn University, Bangkok, Thailand

Introduction As risk behaviours, including unhealthy diet and nutrition, tobacco and alcohol consumption, relevant to noncommunicable chronic diseases are common to oral disease. This study aimed to quantify the extent to which socioeconomic-related inequality in oral health risk behaviours among Thai adolescents is manifested.

Methods Data of 5819 and 4429 Thai adolescents aged 11-19 years living in urban and rural, respectively from the nationally representative Health and Welfare Survey 2009 were used. Descriptive analyses were performed to reveal the features of oral health risk behaviours comprising frequency of eating snacks, drinking sweetened beverages, smoking, alcohol drinking. In addition to the use of concentration index (CI) to quantify the extent of socioeconomicrelated inequalities in these behaviours.

Results Reports of eating snacks and drinking sweetened beverages often (ie, more than 5 days per week) of those adolescents of higher socioeconomic-status were more common than their lower socioeconomic-status counterparts. By contrast, smoking and alcohol consumption reveals reverse gradients as these behaviours were more concentrated among those of lower socioeconomic-status than their peers counterparts. The aforementioned findings are corroborated by $\mathrm{CI}$ values that revealed pro-rich inequality in diet behaviours meanwhile the CI indicates pro-poor inequality in smoking and alcohol consumption.

Conclusion This study demonstrated socioeconomic-related inequality in oral health risk behaviours among Thai adolescents is discernable along the entire spectrum of socioeconomic status. The study also indicates population subgroups that should receive consideration for improving oral health risk behaviours which may be enable for integrated approaches in general health promotion strategies. 